\title{
Inequalities of Aleksandrov body
}

Hu Yan ${ }^{1,2^{*}}$ and Jiang Junhua ${ }^{1}$

\footnotetext{
* Correspondence: huyan12@126. com

${ }^{1}$ Department of Mathematics, Shanghai University, Shanghai 200444, China

Full list of author information is available at the end of the article
}

\begin{abstract}
A new concept of $p$-Aleksandrov body is firstly introduced. In this paper, $p$-BrunnMinkowski inequality and $p$-Minkowski inequality on the $p$-Aleksandrov body are established. Furthermore, some pertinent results concerning the Aleksandrov body and the p-Aleksandrov body are presented.
\end{abstract}

2000 Mathematics Subject Classification:

52A20 52A40

Keywords: Aleksandrov body, p-Aleksandrov body, Brunn-Minkowski inequality, Minkowski inequality

\section{Introduction}

The notion of Aleksandrov body was firstly introduced by Aleksandrov to solve Minkowski problem in 1930s in [1]. The Aleksandrov body establishes the relationship between the convex body containing the origin and the positive continuous functions and characterizes the convex body by means of the positive continuous functions. The Aleksandrov body not only be used to solve Minkowski problem but also has a wide range of applications in other areas of Convex Geometric Analysis. Then, the Aleksandrov body is an essential matter in the Brunn-Minkowski theory and plays an important role in Convex Geometric Analysis. In recent years, Ball [2], Gardner [3,4], Lutwak [5-10], Klain [11], Hug [12], Haberl [13], Schneider [14], Stancu [15], Umanskiy [16] and Zhang [17] have given considerable attention to the Brunn-Minkowski theory and their various generalizations.

The purpose of this paper is to study comprehensively the Aleksandrov body, and most importantly, the $L_{p}$ analogues of Aleksandrov body become a major goal. Here, a new geometric body is firstly introduced, called $p$-Aleksandrov body. Meanwhile, $p$-Brunn-Minkowski inequality and $p$-Minkowski inequality for the $p$-Aleksandrov bodies associated with positive continuous functions are established. Furthermore, some related results, including of the uniqueness results, the convergence results for the Aleksandrov bodies and the $p$-Aleksandrov bodies associated with positive continuous functions, are presented.

Let $\mathcal{K}^{n}$ denote the set of convex bodies (compact, convex subsets with non-empty interiors) in Euclidean space $\mathbb{R}^{n}, \mathcal{K}_{0}^{n}$ denote the set of convex bodies containing the origin in their interiors. Let $V(K)$ denote the $n$-dimensional volume of body $K$, for the standard unit ball $B$ in $\mathbb{R}^{n}$, denote $\omega_{n}=V(B)$, and let $S^{n-1}$ denote the unit sphere in $\mathbb{R}^{n}$.

Let $C^{+}\left(S^{n-1}\right)$ denote the set of positive continuous functions on $S^{n-1}$, endowed with the topology derived from the max norm. Given a function $f \in C^{+}\left(S^{n-1}\right)$, the set 


$$
\left\{K \in \mathcal{K}_{0}^{n}: h_{K} \leq f\right\}
$$

has a unique maximal element, then we denoted the Aleksandrov body associated with the function $f \in C^{+}\left(S^{n-1}\right)$ by

$$
K(f)=\max \left\{K \in \mathcal{K}_{0}^{n}: h_{K} \leq f\right\} .
$$

The volume of body $K(f)$ is denoted by $V(K(f))$. Following Aleksandrov (see [18]), define the volume $V(f)$ of a function $f$ as the volume of the Aleksandrov body associated with the positive continuous function $f$.

In this paper, we generalize and improve Brunn-Minkowski inequality and Minkowski inequality for the Aleksandrov bodies associated with positive continuous functions and establish $p$-Minkowski inequality and $p$-Brunn-Minkowski inequality for the Aleksandrov bodies and the $p$-Aleksandrov bodies associated with positive continuous functions as follows.

\section{Theorem 1}

If $Q \in \mathcal{K}_{0}^{n}, f \in C^{+}\left(S^{n-1}\right)$, and $p \geq 1$, then

$$
V_{p}(Q, f) \geq V(Q)^{(n-p) / n} V(f)^{p / n}
$$

with equality if and only if there exists a constant $c>0$ such that $h_{Q}=c f$, almost everywhere with respect to $S(Q, \cdot)$ on $S^{n-1}$.

\section{Theorem 2}

If $p \geq 1, f, g \in C^{+}\left(S^{n-1}\right)$, and $\lambda, \mu \in \mathbb{R}^{+}$, then

$$
V\left(\lambda \cdot f{ }_{+} \mu \cdot g\right)^{\frac{p}{n}} \geq \lambda V(f)^{\frac{p}{n}}+\mu V(g)^{\frac{p}{n}},
$$

with equality if and only if there exists a constant $c>0$ such that $f=c g$, almost everywhere with respect to $S(K(f), \cdot)$ on $S^{n-1}$.

The other aim of this paper is to establish the following inequality for the Aleksandrov bodies and the $p$-Aleksandrov bodies associated with positive continuous functions.

\section{Theorem 3}

If $K(f), K(g) \in \mathcal{K}_{e}^{n}$, are the Aleksandrov bodies associated with the functions $f, g \in C^{+}\left(S^{n-1}\right)$, and $n \neq p \geq 1$, then

$$
V\left(f+{ }_{p} g\right)^{\frac{n-p}{n}} \geq V(f)^{\frac{n-p}{n}}+V(g)^{\frac{n-p}{n}},
$$

with equality if and only if there exists a constant $c>0$ such that $f=c g$, almost everywhere with respect to $S\left(K(f)\right.$, , ) on $S^{n-1}$.

More interrelated notations, definitions, and their background materials are exhibited in the next section.

\section{Definition and notation}

The setting for this paper is $n$-dimensional Euclidean space $\mathbb{R}^{n}$. Let $\mathcal{K}^{n}$ denote the set of convex bodies (compact, convex subsets with non-empty interiors), $\mathcal{K}_{0}^{n}$ denote the subset of $\mathcal{K}^{n}$ that contains the origin in their interiors, and $\mathcal{K}_{e}^{n}$ denote the subset of $\mathcal{K}^{n}$ 
that are centered in $\mathbb{R}^{n}$. We reserve the letter $u$ for unit vector and the letter $B$ for the unit ball centered at the origin. The surface of $B$ is $S^{n-1}$, and the volume of $B$ denotes $\omega_{n}$.

For $\phi \in G L(n)$, let $\phi^{t}, \phi^{-1}$, and $\phi^{-t}$, denote the transpose, inverse, and inverse of the transpose of $\phi$, respectively. If $K \in \mathcal{K}^{n}$, the support function of $K, h_{K}=h(K, \cdot): \mathbb{R}^{n} \rightarrow$ $(0, \infty)$, is defined by

$$
h(K, u)=\max \{u \cdot x: x \in K\}, u \in S^{n-1},
$$

where $u \cdot x$ denotes the standard inner product of $u$ and $x$.

The set $\mathcal{K}^{n}$ will be viewed as equipped with the usual Hausdorff metric, $d$, defined by $d(K, L)=\left|h_{K}-h_{L}\right|_{\infty}$, where $|\cdot|_{\infty}$ is the sup (or max) norm on the space of continuous functions on the unit sphere, $C\left(S^{n-1}\right)$.

For $K, L \in \mathcal{K}^{n}$, and $\alpha, \beta \geq 0$ (not both zero), the Minkowski linear combination, $\alpha K$ $+\beta L \in \mathcal{K}^{n}$ is defined by

$$
h(\alpha K+\beta L, \cdot)=\alpha h(K, \cdot)+\beta h(L, \cdot) .
$$

Firey introduced, for each real $p \geq 1$, new linear combinations of convex bodies: For $K, L \in \mathcal{K}_{0}^{n}$, and $\alpha, \beta \geq 0$ (not both zero), the Firey combination, $\alpha \cdot K{ }_{{ }_{p}} \beta \cdot L \in \mathcal{K}_{0}^{n}$ whose support function is defined by (see [19])

$$
h\left(\alpha \cdot K+_{p} \beta \cdot L_{r} \cdot\right)^{p}=\alpha h(K, \cdot)^{p}+\beta h\left(L_{,} \cdot\right)^{p} .
$$

Obviously, $\alpha \cdot K=\alpha^{1 / p} K$.

For $K, L \in \mathcal{K}^{n}$, and $\alpha, \beta \geq 0$ (not both zero), by the Minkowski existence theorem (see $[3,14]$ ), there exists a convex body $\alpha \cdot K+\beta \cdot L \in \mathcal{K}^{n}$, such that

$$
S\left(\alpha \cdot K+\beta \cdot L_{,} \cdot\right)=\alpha S(K, \cdot)+\beta S(L, \cdot),
$$

where $S(K, \cdot)$ denotes the surface area measure of $K$, and the linear combination $\alpha$. $K+\beta \cdot L$ is called a Blaschke linear combination.

Lutwak generalized the notion of Blaschke linear combination in [5]:

For $K, L \in \mathcal{K}_{e}^{n}$, and $n \neq p \geq 1$, define $K+_{p} L \in \mathcal{K}_{e}^{n}$ by

$$
S_{p}\left(K+{ }_{p} L, \cdot\right)=S_{p}(K, \cdot)+S_{p}(L, \cdot) .
$$

The existence and uniqueness of $K+_{p} L$ are guaranteed by Minkowski's existence theorem in [5].

\subsection{Mixed volume and $p$-mixed volume}

If $K_{i} \in \mathcal{K}^{n}(\mathrm{I}=1,2, \ldots, r)$ and $\lambda_{i}(i=1,2, \ldots, r)$ are nonnegative real numbers, then of fundamental importance is the fact that the volume of $\sum_{i=1}^{r} \lambda_{i} K_{i}$ is a homogeneous polynomial in $\lambda_{i}$ given by

$$
V\left(\sum_{i=1}^{r} \lambda_{i} K_{i}\right)=\sum_{i_{1}, \ldots, i_{n}} \lambda_{i_{1}} \ldots \lambda_{i_{n}} V\left(K_{i_{1}} \ldots K_{i_{n}}\right),
$$

where the sum is taken over all $n$-tuples $\left(i_{1}, \ldots i_{n}\right)$ of positive integers not exceeding $r$. The coefficient $V\left(K_{i_{1}} \ldots K_{i_{n}}\right)$, which is called the mixed volume of $K_{i_{1}} \ldots K_{i_{n}}$, depends only on the bodies $K_{i_{1}} \ldots K_{i_{n}}$ and is uniquely determined by (2.5). If $K_{1}=\ldots K_{n-i}=K$ and $K_{n-i}$ ${ }_{+1}=\ldots=K_{n}=L$, then the mixed volume $V\left(K_{1} \ldots K_{n}\right)$ is usually written as $V_{i}(K, L)$. 
Let $r=1$ in (2.5), we see that

$$
V\left(\lambda_{1} K_{1}\right)=\lambda_{1}^{n} V\left(K_{1}\right) .
$$

Further, from (2.5), it follows immediately that

$$
n V_{1}(K, L)=\lim _{\varepsilon \rightarrow 0} \frac{V(K+\varepsilon L)-V(K)}{\varepsilon} .
$$

Aleksandrov (see [1]) and Fenchel and Jessen (see [20]) have shown that corresponding to each $K \in \mathcal{K}^{n}$, there is a positive Borel measure, $S(K, \cdot)$ on $S^{n-1}$, called the surface area measure of $K$, such that

$$
V_{1}(K, Q)=\frac{1}{n} \int_{S^{n-1}} h(Q, u) \mathrm{d} S(K, u),
$$

for all $Q \in \mathcal{K}^{n}$.

For $p \geq 1$, the $p$-mixed volume $V_{p}(K, L)$ of $K, L \in \mathcal{K}_{0}^{n}$, was defined by (see [5])

$$
\frac{n}{p} V_{p}(K, L)=\lim _{\varepsilon \rightarrow 0} \frac{V\left(K+_{p} \varepsilon \cdot L\right)-V(K)}{\varepsilon} .
$$

That the existence of this limit was demonstrated in [5].

It was also shown in [5], that corresponding to each $K \in \mathcal{K}_{0}^{n}$, there is a positive Borel measure, $S_{p}(K, \cdot)$ on $S^{n-1}$ such that

$$
V_{p}(K, Q)=\frac{1}{n} \int_{S^{n-1}} h(Q, u)^{p} \mathrm{~d} S_{p}(K, u),
$$

for all $Q \in \mathcal{K}_{0}^{n}$. It turns out that the measure $S_{p}(K, \cdot)$ is absolutely continuous with respect to $S(K, \cdot)$ and has Radon-Nikodym derivative,

$$
\frac{\mathrm{d} S_{p}(K, \cdot)}{\mathrm{d} S(K, \cdot)}=h(K, \cdot)^{1-p} .
$$

From (2.7) and (2.8), we have

$$
V_{p}(K, Q)=\frac{1}{n} \int_{S^{n-1}} h(Q, u)^{p} h(K, u)^{1-p} \mathrm{~d} S(K, u),
$$

where $S(K, \cdot)=S_{0}(K, \cdot)$ is the surface area measure of $K$.

Obviously, for each $K \in \mathcal{K}_{0}^{n}, p \geq 1$,

$$
V_{p}(K, K)=V(K) .
$$

\subsection{Aleksandrov body}

If a function $f \in C^{+}\left(S^{n-1}\right)$ (denoted the set of positive continuous functions on $S^{n-1}$ and endowed with the topology derived from the max norm), the set

$$
\left\{K \in \mathcal{K}_{0}^{n}: h_{K} \leq f\right\}
$$

has a unique maximal element, then the Aleksandrov body associated with the function $f$ is denoted by 


$$
K(f)=\max \left\{K \in \mathcal{K}_{0}^{n}: h_{K} \leq f\right\} .
$$

From (2.11) and (2.1), we have: If $f, g \in C+\left(S^{n-1}\right)$, and $\lambda, \mu \geq 0$ (not both zero), then

$$
K(\lambda f+\mu g) \supseteq \lambda K(f)+\mu K(g) .
$$

Obviously, if $f$ is the support function of a convex body $K$, then the Aleksandrov body associated with $f$ is $K . V(K(f))$ denotes the volume of body $K(f)$. Following Aleksandrov (see [18]), define the volume $V(f)$ of a function $f$ as the volume of the Aleksandrov body associated with the positive function $f$.

For $Q \in \mathcal{K}_{0}^{n}, f \in C^{+}\left(S^{n-1}\right)$, and $p \geq 1, V_{p}(Q, f)$ is defined by (see [5])

$$
V_{p}(Q, f)=\frac{1}{n} \int_{S^{n-1}} f(u)^{p} h(Q, u)^{1-p} \mathrm{~d} S(Q, u),
$$

Obviously, $V_{p}\left(K, h_{K}\right)=V(K)$, for all $K \in \mathcal{K}_{0}^{n}$.

\section{$2.3 p$-Aleksandrov body}

\section{Definition 1}

Let $f, g \in C^{+}\left(S^{n-1}\right), p \geq 1$, and

$$
\varepsilon>-\min \left\{f(u)^{p} / g(u)^{p}, u \in S^{n-1}\right\},
$$

define

$$
f+{ }_{p} \varepsilon \cdot g=\left(f^{p}+\varepsilon g^{p}\right)^{1 / p} .
$$

Then, the set

$$
\left\{Q \in \mathcal{K}_{0}^{n}: h(Q, \cdot) \leq\left(f^{p}+g^{p}\right)^{1 / p}\right\},
$$

has a unique maximal element.

We denote the $p$-Aleksandrov body associated with the function $f+_{p} g \in C^{+}\left(S^{n-1}\right)$ by

$$
K_{p}\left(f+{ }_{p} g\right)=\max \left\{Q \in \mathcal{K}_{0}^{n}: h(Q, \cdot) \leq\left(f^{p}+g^{p}\right)^{1 / p}\right\},
$$

for $p \geq 1$.

The volume of body $K_{p}\left(f+_{p} g\right)$ is denoted by $V\left(K_{p}\left(f+_{p} g\right)\right)$, and define the volume $V\left(f+_{p} g\right)$ of the function $f+_{p} g$ as the volume of the $p$-Aleksandrov body associated with the positive function $f+_{p} g$.

From (2.2), we have the following result: If $f, g \in C^{+}\left(S^{n-1}\right)$, and $p \geq 1$, then

$$
K_{p}\left(f+{ }_{p} g\right) \supseteq K(f)+{ }_{p} K(g) .
$$

We note that the equality condition in (2.16) is clearly holds, when $f$ and $g$ are the support functions of $K(f)$ and $K(g)$, respectively. Also, the case $p=1$ of (2.16) is just (2.12).

\section{Proof of the main results}

The following Lemmas will be required to prove our main theorems.

\section{Lemma 1}

[5]If $K(f)$ is the Aleksandrov body associated with $f \in C^{+}\left(S^{n-1}\right)$, then $h_{K(f)}=f$ almost everywhere with respect to the measure $S\left(K(f)\right.$, , ) on $S^{n-1}$. 
Obviously, if $K(f)$ is the Aleksandrov body corresponding to a given function $f \in C$ ${ }^{+}\left(S^{n-1}\right)$, its support function has the property that $0<h_{K} \leq f$ and $V(f)=V\left(h_{K(f)}\right)$.

\section{Lemma 2}

[5]If $p \geq 1, K(f)$ is the Aleksandrov body associated with $f \in C^{+}\left(S^{n-1}\right)$, then $V(f)=V$ $(K(f))=V_{p}(K(f), f)$, i.e

$$
V(f)=\frac{1}{n} \int_{S^{n-1}} h(K(f), u) \mathrm{d} S(K(f), u) .
$$

\section{Lemma 3}

[5]If $K \in \mathcal{K}_{0}^{n}, f \in C^{+}\left(S^{n-1}\right)$, then, for $p \geq 1$,

$$
\frac{n}{p} V_{p}(K, f)=\lim _{\varepsilon \rightarrow 0} \frac{V\left(h_{K}+{ }_{p} \varepsilon \cdot f\right)-V\left(h_{K}\right)}{\varepsilon} .
$$

We get the following Brunn-Minkowski inequality for the Aleksandrov bodies associated with positive continuous functions.

\section{Lemma 4}

If $f, g \in C^{+}\left(S^{n-1}\right)$, and $\lambda, \mu \in \mathbb{R}^{+}$, then

$$
V(\lambda f+\mu g)^{1 / n} \geq \lambda V(f)^{1 / n}+\mu V(g)^{1 / n},
$$

with equality if and only if there exist a constant $c>0$ and $t \geq 0$, such that $f=c g+t$, almost everywhere with respect to $S(K(f),$.$) on S^{n-1}$.

Proof

Since $f, g \in C^{+}\left(S^{n-1}\right)$, from (2.11), (2.12) and the Brunn-Minkowski inequality (see [21]), we get

$$
\begin{aligned}
V(K(\lambda f+\mu g))^{1 / n} & \geq V(\lambda K(f)+\mu K(g))^{1 / n} \\
& \geq \lambda V(K(f))^{1 / n}+\mu V(K(g))^{1 / n} .
\end{aligned}
$$

The equality condition in (3.3) is that $f, g$ are the support functions of $K(f)$ and $K(g)$ which are homothetic, respectively.

From Lemma 1 and Lemma 2, we get the following result

$$
V(\lambda f+\mu g)^{1 / n} \geq \lambda V(f)^{1 / n}+\mu V(g)^{1 / n},
$$

with equality if and only if there exist a constant $c>0$ and $t \geq 0$, such that $f=c g+t$, almost everywhere with respect to $S(K(f), \cdot)$ on $S^{n-1}$.

An immediate consequence of the definition of a Firey linear combination, and the integral representation (2.13), is that for $Q \in \mathcal{K}_{0}^{n}$, the $p$-mixed volume

$$
V_{p}(Q, \cdot): C^{+}\left(S^{n-1}\right) \rightarrow(0, \infty)
$$

is Firey linear. 


\section{Lemma 5}

If $p \geq 1, Q \in \mathcal{K}_{0}^{n}, f, g \in C^{+}\left(S^{n-1}\right)$, and $\lambda, \mu \in \mathbb{R}^{+}$, then

$$
V_{p}\left(Q, \lambda \cdot f+_{p} \mu \cdot g\right)=\lambda V_{p}(Q, f)+\mu V_{p}(Q, g) \text {. }
$$

\section{Proof}

From (2.13), (2.14), we obtain

$$
\begin{aligned}
V_{p}\left(Q, \lambda \cdot f+_{p} \mu \cdot g\right) & =\frac{1}{n} \int_{S^{n-1}}\left(\lambda \cdot f+_{p} \mu \cdot g\right)^{p} h(Q, u)^{1-p} \mathrm{~d} S(Q, u) \\
& =\frac{1}{n} \int_{S^{n-1}}\left(\lambda f^{p}+\mu g^{p}\right) h(Q, u)^{1-p} \mathrm{~d} S(Q, u) \\
& =\lambda V_{p}(Q, f)+\mu V_{p}(Q, g) .
\end{aligned}
$$

In the following, we will prove the $p$-Minkowski inequality for the Aleksandrov bodies associated with positive continuous functions.

Proof of Theorem 1.

Firstly, let $p=1$ in Lemma 3, we get

$$
n V_{1}(Q, f)=\lim _{\varepsilon \rightarrow 0} \frac{V\left(h_{Q}+\varepsilon f\right)-V\left(h_{Q}\right)}{\varepsilon},
$$

let $\varepsilon=\frac{t}{1-t}$, we have

$$
\begin{aligned}
n V_{1}(Q, f) & =\lim _{t \rightarrow 0} \frac{V\left((1-t) h_{Q}+t f\right)-(1-t)^{n} V\left(h_{Q}\right)}{t(1-t)^{n-1}} \\
& =\lim _{t \rightarrow 0} \frac{V\left((1-t) h_{Q}+t f\right)-V\left(h_{Q}\right)}{t}+\lim _{t \rightarrow 0} \frac{\left(1-(1-t)^{n}\right) V\left(h_{Q}\right)}{t} \\
& =\lim _{t \rightarrow 0} \frac{V\left((1-t) h_{Q}+t f\right)-V\left(h_{Q}\right)}{t}+n V\left(h_{Q}\right) .
\end{aligned}
$$

Let

$$
f(t)=V\left((1-t) h_{Q}+t f\right)^{1 / n}, \quad 0 \leq t \leq 1,
$$

we see that

$$
f^{\prime}(0)=\frac{V_{1}(Q, f)-V\left(h_{Q}\right)}{V\left(h_{Q}\right)^{\frac{n-1}{n}}} .
$$

From Lemma 4, we know that $f$ is concave, i.e.

$$
\frac{V_{1}(Q, f)-V\left(h_{Q}\right)}{V\left(h_{Q}\right)^{\frac{n-1}{n}}} \geq V(f)^{\frac{1}{n}}-V\left(h_{Q}\right)^{\frac{1}{n}} .
$$

Thus,

$$
V_{1}(Q, f) \geq V(Q)^{\frac{n-1}{n}} V(f)^{\frac{1}{n}}
$$

According to the equality condition in inequality (3.3), and using Lemma 1 and Lemma 2, we have the equality holds in inequality (3.6), if and only if there exist a 
constant $c>0$ and $t \geq 0$, such that $h_{Q}=c f+t$, almost everywhere with respect to $S$ $(Q, \cdot)$ on $S^{n-1}$.

Secondly, from the Hölder inequality (see [22]), together with the integral representations (2.13) and (2.6), we obtain

$$
\begin{aligned}
V_{p}(Q, f) & =\frac{1}{n} \int_{S^{n-1}} f(u)^{p} h(Q, u)^{1-p} \mathrm{~d} S(Q, u) \\
& \geq V_{1}(Q, f)^{p} V(Q)^{1-p},
\end{aligned}
$$

when this combined with inequality (3.6), we have

$$
V_{p}(Q, f) \geq V(Q)^{\frac{n-p}{n}} V(f)^{\frac{p}{n}} .
$$

To obtain the equality conditions, we note that there is equality in Hölder's inequality precisely when $V_{1}(Q, f) h_{Q}=V(Q) f$, almost everywhere with respect to the measure $S(Q, \cdot)$ on $S^{n-1}$. Combining the equality conditions in (3.6), and using Lemma 1 , it shows that the equality holds if and only if there exists a constant $c>0$ such that $h_{Q}=$ $c f$, almost everywhere with respect to $S(Q, \cdot)$ on $S^{n-1}$.

Using the above Lemmas and Theorem 1, we can get the following Corollaries describing the uniqueness results.

\section{Corollary 1}

Suppose $K, L \in \mathcal{K}_{0}^{n}$, and $\mathcal{F} \subset C^{+}\left(S^{n-1}\right)$ is a class of functions such that $h_{K}, h_{L} \in \mathcal{F}$. (i) If $n \neq p>1$, and $V_{p}(K, f)=V_{p}(L, f)$, for all $f \in \mathcal{F}$, then $K=L$. (ii) If $p=n$, and $V_{p}(K, f)$ $\geq V_{p}(L, f)$, for all $f \in \mathcal{F}$, then $K$ and $L$ are dilates, and hence

$$
V_{p}(K, f)=V_{p}(L, f), \text { for all } f \in C^{+}\left(S^{n-1}\right) .
$$

\section{Proof}

If $n \neq p>1$, take $f=h_{K}$, and from (2.13), Lemma 2 and Theorem 1, we get

$$
V_{p}(K, f)=V_{p}\left(K, h_{K}\right)=V_{p}\left(L, h_{K}\right) \geq V(L)^{\frac{n-p}{n}} V\left(h_{K}\right)^{\frac{p}{n}} .
$$

Hence,

$$
V(K) \geq V(L) .
$$

Similarly, take $f=h_{L}$, we get

$$
V(L) \geq V(K) .
$$

In view of the equality conditions of Theorem 1 , we obtain that $K=L$.

If $n=p$, the hypothesis together with Theorem 1, we have

$$
V_{p}(K, f) \geq V_{p}(L, f) \geq V(L)^{\frac{n-p}{n}} V(f)^{\frac{p}{n}},
$$

with equality in the right inequality implying that $L$ and $K(f)$ are dilates. Take $f=h_{K}$, since $n=p$, the terms on the left and right are identical, and thus, $K$ and $L$ must dilates; hence,

$$
V_{p}(K, f)=V_{p}(L, f), \quad \text { for all } f \in C^{+}\left(S^{n-1}\right) .
$$




\section{Corollary 2}

Suppose $f, g \in C^{+}\left(S^{n-1}\right)$, and $\mathcal{F} \subset C^{+}\left(S^{n-1}\right)$ is a class of functions such that $f, g \in \mathcal{F}$. If $p$ $>1$, and

$$
V_{p}(Q, f)=V_{p}(Q, g), \quad \text { for all } h_{Q} \in \mathcal{F},
$$

then $f=g$ almost everywhere on $S^{n-1}$.

\section{Proof}

Since $f, g \in C^{+}\left(S^{n-1}\right)$, according to (2.11), we denote two Aleksandrov bodies $K(f)$ and $K(g)$. From the hypothesis, taking $Q=K(f)$, and using Lemma 2 and Theorem 1, we get

$$
V_{p}(K(f), f)=V(f)=V_{p}(K(f), g) \geq V(K(f))^{\frac{n-p}{n}} V(g)^{\frac{p}{n}},
$$

then,

$$
V(f) \geq V(g) .
$$

Similarly, take $Q=K(g)$, we get

$$
V(g) \geq V(f) .
$$

From the equality conditions of Theorem 1 , we obtain

$$
K(f)=K(g) .
$$

In view of the definition of Aleksandrov body, and using Lemma 1, then

$$
f=g, \quad \text { almost everywhere on } S^{n-1} \text {. }
$$

\section{Corollary 3}

Suppose $n \neq p>1$, and $f, g \in C^{+}\left(S^{n-1}\right)$, such that $S_{p}(K(f), \cdot) \leq S_{p}(K(g), \cdot)$.

(i) If $V(f) \geq V(g)$, and $p<n$, then $f=g$ almost everywhere on $\mathrm{S}^{n-1}$.

(ii) If $V(f) \leq V(g)$, and $p>n$, then $f=g$ almost everywhere on $S^{n-1}$.

\section{Proof}

Suppose a function $\phi \in C^{+}\left(S^{n-1}\right)$, and $n \neq p>1$, since $S_{p}(K(f), \cdot) \leq S_{p}(K(g), \cdot)$, it follows from the integral representation (2.13) and (2.8) that

$$
V_{p}(K(f), \phi) \leq V_{p}(K(g), \phi) \text {, for all } \phi \in C^{+}\left(S^{n-1}\right) .
$$

As before, take $\phi=h_{K(g)}$, from Lemma 1, Lemma 2, and Theorem 1, we get

$$
V(f)^{\frac{n-p}{n}} \leq V(g)^{\frac{n-p}{n}} .
$$

Applying the hypothesis, and from the definition of the Aleksandrov body and Lemma 1, we obtain the desired results.

\section{Corollary 4}

Suppose $n \neq p \geq 1, f, g \in C^{+}\left(S^{n-1}\right)$, and $\mathcal{F} \subset C^{+}\left(S^{n-1}\right)$ is a class of functions such that $f$, $g \in \mathcal{F}$. If 


$$
\frac{V_{p}(K, f)}{V(f)}=\frac{V_{p}(K, g)}{V(g)}, \text { for all } h_{K} \in \mathcal{F},
$$

then $f=g$ almost everywhere on $S^{n-1}$.

\section{Proof}

According to (2.11), we denote two Aleksandrov bodies $K(f)$ and $K(g)$. From the hypothesis, taking $K=K(f)$ and $K=K(g)$, and combining with Lemma 2 and Theorem 1 , respectively, we obtain

$$
V(g) \geq V(f) \quad \text { and } \quad V(f) \geq V(g),
$$

Hence, in view of the equality conditions of Theorem 1, the definition of Aleksandrov body, and Lemma 1, we get the desired result

$$
f=g, \quad \text { almost everywhere on } S^{n-1} .
$$

Now, the $p$-Brunn-Minkowski inequality for the $p$-Aleksandrov bodies and the Aleksandrov bodies associated with positive continuous functions is established as following.

Proof of Theorem 2.

From Lemma 5 and Theorem 1, we get

$$
\begin{aligned}
V_{p}\left(Q, \lambda \cdot f_{+_{p}} \mu \cdot g\right) & =\lambda V_{p}(Q, f)+\mu V_{p}(Q, g) \\
& \geq V(Q)^{\frac{n-p}{n}}\left[\lambda V(f)^{\frac{p}{n}}+\mu V(g)^{\frac{p}{n}}\right],
\end{aligned}
$$

with equality if and only if $K(f)$ and $K(g)$ are dilates of $Q$.

Now, take $Q=K_{p}\left(\lambda \cdot f+_{p} \mu \cdot g\right)$, use (2.10), and recall $V(f)=V(K(f))=V_{p}(K(f), f)$, we have

$$
V\left(\lambda \cdot f{ }_{+} \mu \cdot g\right)^{\frac{p}{n}} \geq \lambda V(f)^{\frac{p}{n}}+\mu V(g)^{\frac{p}{n}} .
$$

Also, we note that the equality holds, if and only if $K(f)$ and $K(g)$ are dilates. Using Lemma 1, we get the condition of equality holds if and only if there exists a constant $c$ $>0$ such that $f=c g$, almost everywhere with respect to $S(K(f), \cdot)$ on $S^{n-1}$.

Then, we will prove Theorem 3 by using the generalized Blaschke linear combination.

\section{Proof of Theorem 3.}

Suppose a function $\phi \in C^{+}\left(S^{n-1}\right)$, and $n \neq p \geq 1$, from the integral representation (2.13), (2.8), and (2.4), it follows that for $K(f), K(g) \in \mathcal{K}_{e}^{n}$,

$$
V_{p}\left(K(f)+{ }_{p} K(g), \phi\right)=V_{p}(K(f), \phi)+V_{p}(K(g), \phi),
$$

which together with Theorem 1, yields

$$
V_{p}\left(K(f)+{ }_{p} K(g), \phi\right) \geq V(\phi)^{\frac{p}{n}}\left[V(K(f))^{\frac{n-p}{n}}+V(K(g))^{\frac{n-p}{n}}\right],
$$

with equality if and only if $K(f) ; K(g)$ and $K(\phi)$ are dilates.

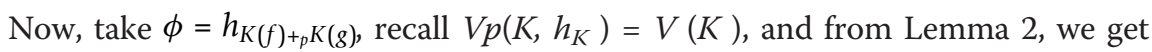

$$
V\left(K(f)+{ }_{p} K(g)\right)^{\frac{n-p}{n}} \geq V(f)^{\frac{n-p}{n}}+V(g)^{\frac{n-p}{n}} .
$$


In view of (2.16), we have

$$
V\left(K_{p}(f+p g)\right) \geq V\left(K(f)+_{p} K(g)\right) .
$$

Hence, we get

$$
\begin{aligned}
V\left(K_{p}(f+p g)\right)^{\frac{n-p}{n}} & \geq V\left(K(f)+{ }_{p} K(g)\right)^{\frac{n-p}{n}} \\
& \geq V(f)^{\frac{n-p}{n}}+V(g)^{\frac{n-p}{n}} .
\end{aligned}
$$

From Lemma 2 again, we obtain

$$
V(f+p g)^{\frac{n-p}{n}} \geq V(f)^{\frac{n-p}{n}}+V(g)^{\frac{n-p}{n}} .
$$

In view of the equality condition (3.9), and from Lemma 1, we get the equality holds if and only if there exists a constant $c>0$ such that $f=c g$, almost everywhere with respect to $S(K(f), \cdot)$ on $S^{n-1}$.

\section{Remark 1}

The case $p=1$ of the inequality of Theorem 3 is

$$
V(f+g)^{\frac{n-1}{n}} \geq V(f)^{\frac{n-1}{n}}+V(g)^{\frac{n-1}{n}},
$$

with equality if and only if there exists a constant $c>0$ such that $f=c g$, almost everywhere with respect to $S(K(f), \cdot)$ on $S^{n-1}$.

The above inequality (3.10) is just the Kneser-Süss inequality type for the Aleksandrov bodies associated with positive continuous functions.

Actually, from these above proofs, we see Brunn-Minkowski inequality, Minkowski inequality, and Knesser-Süss inequality are equivalent.

\section{Convergence of Aleksandrov body}

In this section, we establish a convergent result about the Aleksandrov bodies associated with positive continuous functions.

The following Lemmas will be required to prove our main result.

\section{Lemma 6}

[7]If $p \geq 1$, and $K_{i}$ is a sequence of bodies in $\mathcal{K}_{0}^{n}$, such that $K_{i} \rightarrow K_{0} \in \mathcal{K}_{0}^{n}$, then $S_{p}\left(K_{i}, \cdot\right)$ $\rightarrow S_{p}\left(K_{0}, \cdot\right)$, weakly.

\section{Lemma 7}

Suppose $K_{i} \rightarrow K \in \mathcal{K}_{0}^{n}$, and $f_{i} \rightarrow f \in C^{+}\left(S^{n-1}\right)$. If $p \geq 1$, then $V_{p}\left(K_{i} f_{i}\right) \rightarrow V_{p}(K, f)$.

\section{Proof}

Since $f_{i} \rightarrow f \in C^{+}\left(S^{n-1}\right)$, the $f_{i}$ are uniformly bounded on $S^{n-1}$. Hence,

$$
f_{i}^{p} \rightarrow f^{p}, \quad \text { uniformly on } S^{n-1} .
$$

By Lemma 6, $K_{i} \rightarrow K$ implies that

$$
S_{p}\left(K_{i}, \cdot\right) \rightarrow S_{p}(K, \cdot), \quad \text { weakly on } S^{n-1} .
$$


Hence,

$$
\int_{S^{n-1}} f_{i}(u)^{p} \mathrm{~d} S_{p}\left(K_{i}, u\right) \rightarrow \int_{S^{n-1}} f(u)^{p} \mathrm{~d} S_{p}(K, u) .
$$

In view of the integral representation (2.13) and (2.8), we get the desired result. The convergence result will be established as following.

\section{Theorem 4}

Suppose $p>1, f \in C^{+}\left(S^{n-1}\right)$. If $f_{i}$ is a sequence of functions in $C^{+}\left(S^{n-1}\right)$, such that

$$
V_{p}\left(Q, f_{i}\right) \rightarrow V_{p}(Q, f), \quad \text { for all } Q \in \mathcal{K}_{0}^{n},
$$

then $f_{i} \rightarrow f$.

\section{Proof}

Firstly, since $f_{i}$ is a sequence in $C^{+}\left(S^{n-1}\right), f_{i}$ are uniformly bounded on $S^{n-1}$.

Applying the Blaschke selection theorem (see [3]), it guarantees the existence of a subsequence of the $f_{i}$, which is again denoted by $f_{i}$, converging to a positive continuous function $f_{0}$ on $S^{n-1}$.

Secondly, since $f_{i}$ are uniformly bounded on $S^{n-1}$,

$$
1+{ }_{p} f_{i} \rightarrow 1+{ }_{p} f_{0}, \quad \text { uniformly on } S^{n-1} .
$$

Define $\bar{f}_{i} \in C^{+}\left(S^{n-1}\right)$, by $\bar{f}_{i}=1+{ }_{p} f_{i}$. Since $\bar{f}_{i} \rightarrow \bar{f}_{0}$, it follows from Lemma 7 that

$$
V_{p}\left(Q, \bar{f}_{i}\right) \rightarrow V_{p}\left(Q, \bar{f}_{0}\right), \text { for all } Q \in \mathcal{K}_{0}^{n} .
$$

However, since $V_{p}\left(Q, f_{i}\right) \rightarrow V_{p}(Q, f)$, for all $Q \in \mathcal{K}_{0}^{n}$, and $\bar{f}_{i}=1{ }_{{ }_{p}} f_{i}$, for all $i>0$, it follows from Lemma 5 that

$$
V_{p}\left(Q, \bar{f}_{i}\right) \rightarrow V_{p}\left(Q, 1+{ }_{p} f\right), \quad \text { for all } Q \in \mathcal{K}_{0}^{n} .
$$

Thus,

$$
V_{p}\left(Q, \bar{f}_{0}\right)=V_{p}\left(Q, 1+{ }_{p} f\right), \quad \text { for all } Q \in \mathcal{K}_{0}^{n} .
$$

By Corollary 2, this means $\bar{f}_{0}=1+{ }_{p} f$, which shows $f_{0}=f$.

Thus, every subsequence of $f_{i}$ has a subsequence that converges to $f$.

\section{Acknowledgements}

The authors express their deep gratitude to the referees for their many very valuable suggestions and comments. The research of Hu-Yan and Jiang-Junhua was supported by National Natural Science Foundation of China (10971128), Shanghai Leading Academic Discipline Project (S30104), and the research of Hu-Yan was partially supported by Innovation Program of Shanghai Municipal Education Commission (10yz160).

\section{Author details}

'Department of Mathematics, Shanghai University, Shanghai 200444, China ²Department of Mathematics, Shanghai University of Electric Power, Shanghai 200090, China

\section{Authors' contributions}

HY and JJH jointly contributed to the main results Theorem 1, Theorem 2, Theorem 3 and Theorem 4. HY drafted the manuscript and made the text file. Both authors read and approved the final manuscript.

\section{Competing interests}

The authors declare that they have no competing interests. 


\section{References}

1. Aleksandrov, AD: On the theory of mixed volumes.I. Extension of certain concepts in the theory of convex bodies. Mat Sb. 2(5), 947-972 (1937)

2. Ball, K: Volume ratios and a Reverse isoperimetric. J Lond Math Soc. 44(1), 351-359 (1991)

3. Gardner, RJ: Geometric Tomography. Cambridge University Press, Cambridge (1995)

4. Gardner, RJ: Intersection bodies and the Busemann-Petty problem. Trans Am Math Soc. 342(1), 435-335 (1994). doi:10.2307/2154703

5. Lutwak, E: The Brunn-Minkowski-Firey Theory I: mixed volume and the Minkowski problem. J Differ Geom. 38(1), 131-150 (1993)

6. Lutwak, E, Oliker, V: On the regularity of solutions to a generalization of the Minkowski problem. J Differ Geom. 41(1), 227-246 (1995)

7. Lutwak, E: The Brunn-Minkowski-Firey Theory II: affine and geominimal surface area. Adv Math. 118(2), $244-294$ (1996) doi:10.1006/aima.1996.0022

8. Lutwak, E, Yang, D, Zhang, G: $L_{p^{-}}$affine isoperimetric inequalities. J Differ Geom. 56(1), 111-132 (2000)

9. Lutwak, E: On the $L_{p}$-Minkowski problem. Trans Am Math Soc. 356(11), 4359-4370 (2003)

10. Lutwak, E, Yang, D, Zhang, G: Optimal Sobolev norms and the $L^{P}$ Minkowski problem. Int Math Res Not. 62987, 1-21 (2006)

11. Klain, DA: The Minkowski problem for polytope. Adv Math. 185(2), 270-288 (2004). doi:10.1016/j.aim.2003.07.001

12. Hug, D, Lutwak, E, Yang, D., et al: On the $L_{p}$ Minkowski problem for polytope. Discret Comput Geom. 33(1), 699-715 (2005)

13. Haberl, C, Lutwak, E, Yang, D., et al: The even Orlicz-Minkowski problem. Adv Math.224(1), 2485-2510

14. Schneider, R: Convex bodies: the Brunn-Minkowski theory. Cambridge University Press, Cambridge (1993)

15. Stancu, A: The discrete planar $L_{0}-$ Minkowski problem. Adv Math. 167(1), 160-174 (2002). doi:10.1006/aima.2001.2040

16. Umanskiy, V: On solvability of two-dimensional $L_{p}$-Minkowski problem. Adv Math. 180(1), 176-186 (2003). doi:10.1016/ S0001-8708(02)00101-9

17. Zhang, GY: Centered bodies and dual mixed volumes. Trans Am Math Soc. 345(1), 777-801 (1994)

18. Aleksandrov, AD: On the theory of mixed volumes.lll. Extension of two theorems of Minkowski on convex polyhedra to arbitrary convex bodies. Mat Sb. 3(1), 27-46 (1938)

19. Firey, WJ: p-means of convex bodies. Math Scand. 10(1), 17-24 (1962)

20. Fenchel, W, Jessen, B: Mengenfunktionen und konvexe Körper. Det Kgl Danske Videnskab Selskab Math-fys Medd. 16(3), 1-31 (1938)

21. Gardner, RJ: The Brunn-Minkowski inequality. Bull Am Math Soc. 39(3), 355-405 (2002). doi:10.1090/S0273-0979-02 $00941-2$

22. Hardy, GH, Littlewood, JE, Pölya, G: Inequalities. Cambridge University Press, Cam-bridge. (1934)

doi:10.1186/1029-242X-2011-39

Cite this article as: Yan and Junhua: Inequalities of Aleksandrov body. Journal of Inequalities and Applications 2011 2011:39.

\section{Submit your manuscript to a SpringerOpen ${ }^{\circ}$} journal and benefit from:

- Convenient online submission

- Rigorous peer review

- Immediate publication on acceptance

- Open access: articles freely available online

- High visibility within the field

- Retaining the copyright to your article

Submit your next manuscript at $>$ springeropen.com 